\title{
Basic processing in cardiovascular MRI: Tips and tricks
}

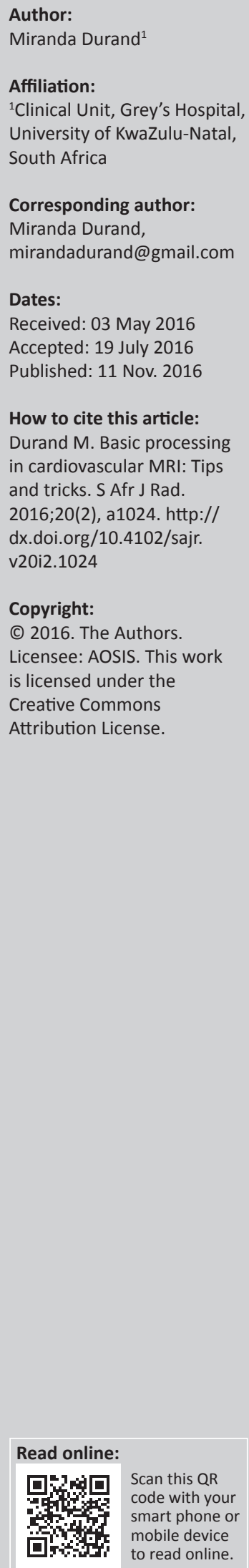

Cardiovascular magnetic resonance (CMR) imaging has gained popularity in the past few years as a reliable, comprehensive assessment tool for the evaluation of patients with congenital and acquired heart disease. However, the overwhelming amount of data generated by CMR makes it difficult to know where to start and what to report. To ensure consistency and reproducibility of CMR reporting, the Society of Cardiovascular Magnetic Resonance (SCMR) Board of Trustees Task Force on Standardised Post Processing has published guidelines to standardise CMR imaging and post processing. The present article aims to provide a pictorial review of these guidelines as well as a framework to basic processing that encompasses: left ventricular function and mass assessment; right ventricular function assessment; atrial size measurements; flow analysis; and T2* analysis and aortic measurements. Cardiac MRI is a robust and rapidly developing field that has significantly advanced the management of cardiac patients. It is important that accuracy and consistency be maintained to ensure that we gain and maintain clinicians' confidence in the use of this modality.

\section{Introduction}

Cardiovascular magnetic resonance (CMR) imaging has gained popularity in the past few years as a reliable, comprehensive assessment tool for the evaluation of patients with congenital and acquired heart disease. A recent review has shown that, compared with modalities such as echocardiography and radionuclide studies, CMR is able to most accurately quantify a patient's clinical condition as well as best predict clinical outcomes across a range of cardiac diseases. ${ }^{1}$

CMR generates many quantitative parameters, and it can be difficult for the beginner to know where to start and what to report. To ensure consistency and reproducibility of CMR reporting, the Society of Cardiovascular Magnetic Resonance (SCMR) Board of Trustees Task Force on Standardised Post Processing has published guidelines to standardise CMR imaging and post processing. ${ }^{2}$

The present article aims to provide a pictorial review of these guidelines as well as a framework on how to approach and interpret the multitude of images acquired by CMR.

The basic CMR processing measurements includes:

- left ventricular (LV) function and mass assessment

- right ventricular (RV) function assessment

- atrial size measurements

- flow analysis

- $\mathrm{T} 2 *$ analysis

- aortic measurements.

More advanced processing such as myocardial tagging, T1 and T2 mapping, as well as diffusion imaging is not covered in the article. ${ }^{3}$

\section{Left ventricular function and mass}

Studies have demonstrated that there is less variance in LV volume and ejection fraction assessment with CMR in comparison with echocardiography and radionuclide ventriculography, which has led to CMR becoming the gold standard for LV function assessment. ${ }^{4}$ However, processing CMR images of LV function can be daunting to the uninitiated and it is useful to have an approach that will guide the beginner through the process. The basic approach is as follows. 


\section{Play the cine movie of the short-axis images first}

This provides an overview of the LV function and wall motion and allows visual assessment of the LV function. Thereafter, correlate this qualitative assessment with the calculated values to ensure that major errors do not occur, such as when the incorrect phase is selected.

Regional wall motion is assessed by using the degree of wall thickening during systole in each LV segment. However, small differences are sometimes difficult to appreciate visually at first glance.

Tip: It helps to put the mouse cursor in the middle of the ventricle as a point to fix the eye upon, and then to assess the degree of LV wall movement towards the cursor (Figure 1).

\section{Delete repeated slices}

Scroll through all the slices, from the basal slices at the atrioventricular (AV) valve to the apex, to ensure that the whole ventricle has been included in the data set. Examine the data set for duplicate slices; this may occur when the patient takes one excessively deep breath hold which then causes the next scanned slice (acquired with a regular breath hold) to overlap with the previous slice. Any repeated slice must be deleted.

Tip: The easiest way to detect a slice that has been repeated is to look for papillary muscles that are in exactly the same position as those in the previous slice (Figure 2).

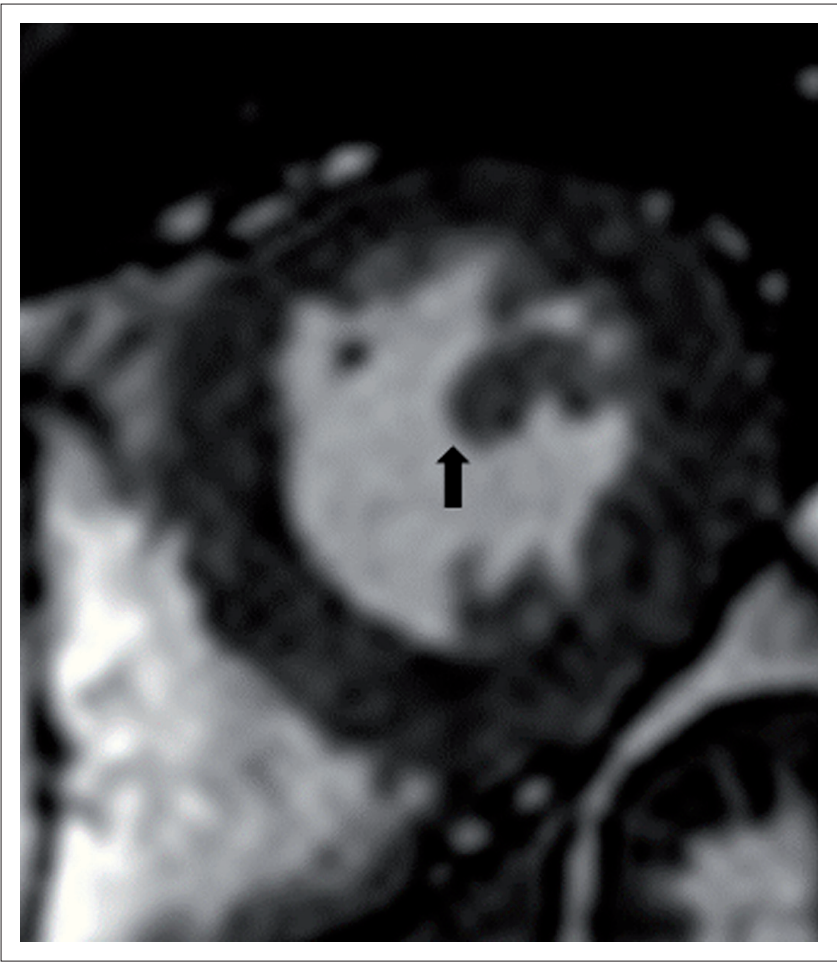

FIGURE 1: Single short-axis cine steady-state free precession (SSFP) image of the LV. The black arrow in the centre of the LV blood pool provides a fixed point to focus the eye upon, which assists in the assessment of the LV wall motion. The degree by which one segment of the LV wall moves towards the arrow (when compared with the rest of the LV segments) simplifies wall motion dysfunction assessment. For video footage, visit https://www.youtube.com/ watch?v=8ljrhHYqOWs\&feature=youtu.be.

\section{Pick the end-systolic and end-diastolic phases}

Most processing software will automatically suggest the end-systolic and end-diastolic phases but the operator still has to check to ensure that it is the correct phase. Arrhythmias may cause the automatic software to select the wrong phase. The end-systolic phase is the phase during the cardiac cycle when the ventricular volume is at its minimum.

Tip: The easiest way to identify end-systole is to examine all the phases at a particular slice level; the mid-ventricular level is normally the easiest to use. End-systole is normally the phase where the myocardium is closest to the papillary muscles (Figure 3).

However, this technique is ineffective in patients with LV dyssynchrony or with severe mitral valve regurgitation. In such cases, the closure of the aortic valve will indicate endsystole. End-diastole is the phase where the ventricular volume is at its maximum and it is normally the phase just before the myocardium starts moving in towards the papillary muscle again.

\section{Deciding on the basal slice for each phase}

The operator selects the most basal slice to use for contouring the end-systolic and end-diastolic phases. This is normally not the same slice for both phases as the mitral annulus moves apically during systole. There are normally one or two slice differences between the basal slice for the endsystolic and end-diastole phases. With severe LV dysfunction, there may be very little apical movement and the basal slice may be the same for end-systole and end-diastole. The recommendation for deciding the most basal slice to be utilised is the one in which at least 180 degrees of myocardium surrounds the LV blood pool (Figure 4).

\section{Contouring the epicardial and endocardial borders}

Tip: It often helps to play the cine movie of the short-axis on a different window while one contours, as this will assist to determine where to draw the endocardial border.

When contouring the endocardial border, the LV outflow tract should be included in the blood pool to the level of the aortic valve (Figure 5). If only myocardium is present in the most basal or most apical slice with no discernible LV blood pool, only an epicardial border is contoured for LV mass assessment (Figure 6). The papillary muscles are not part of the blood pool but, because most entry-level software does not automatically exclude the papillary muscles, and manual contouring of the papillary muscles is extremely labour intensive, the Task Force recommends that the papillary muscles be included or excluded at the operator's preference. Whether including or excluding the papillary muscles, that decision should be reflected in the report and should be consistently applied at any one institution. 

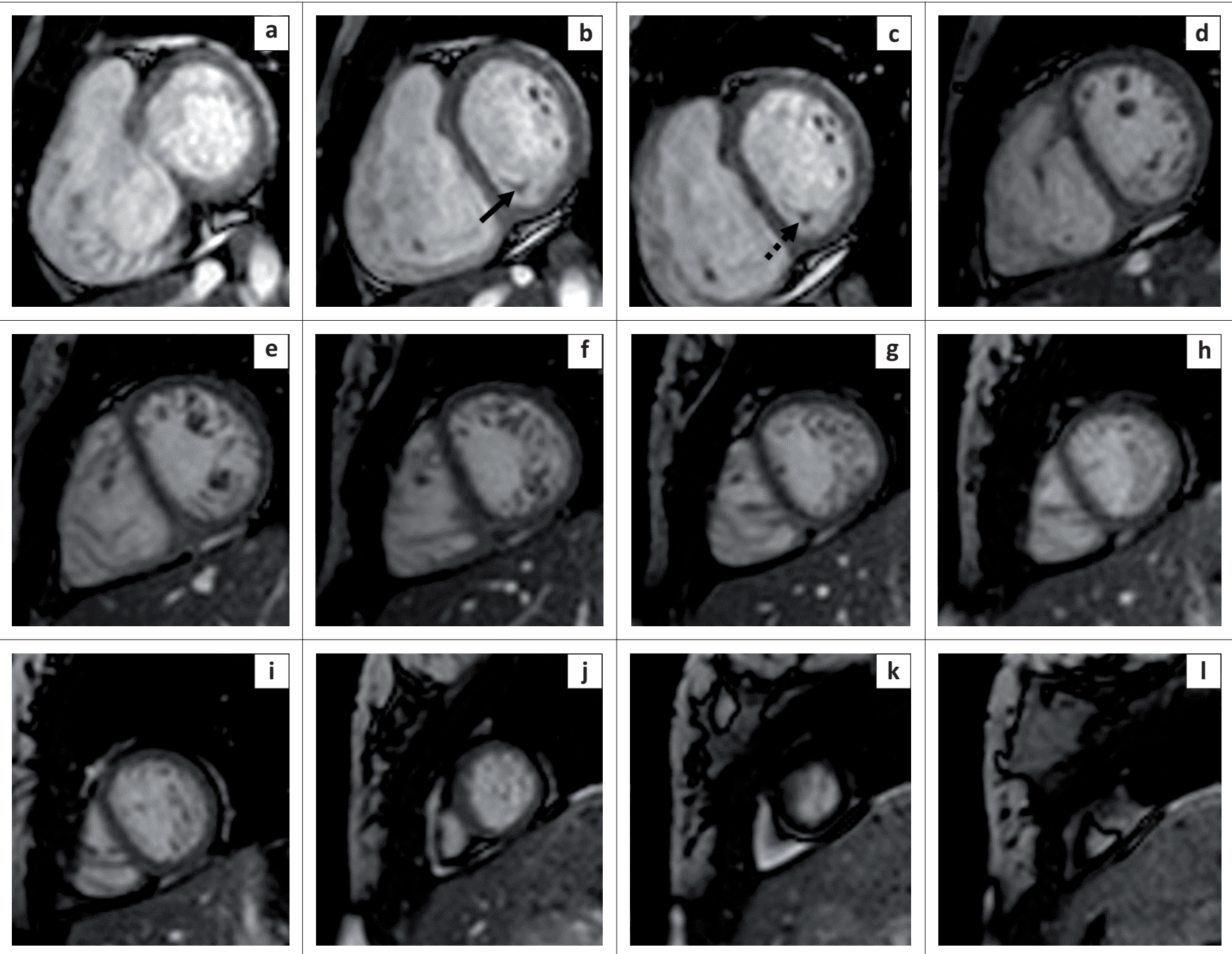

FIGURE 2: A series (a-1) of short-axis cine SSFP images of the LV during a single phase at different slice positions. Slices that repeat the same slice position should be deleted to prevent over-sampling. This is easily determined by observing the configuration of the papillary muscles; the papillary muscle of the second slice (solid black arrow) is in the exact same position and configuration as the papillary muscles of the third slice (stippled black arrow), hence one of these slices must be deleted and not included in the LV volume and mass assessment.
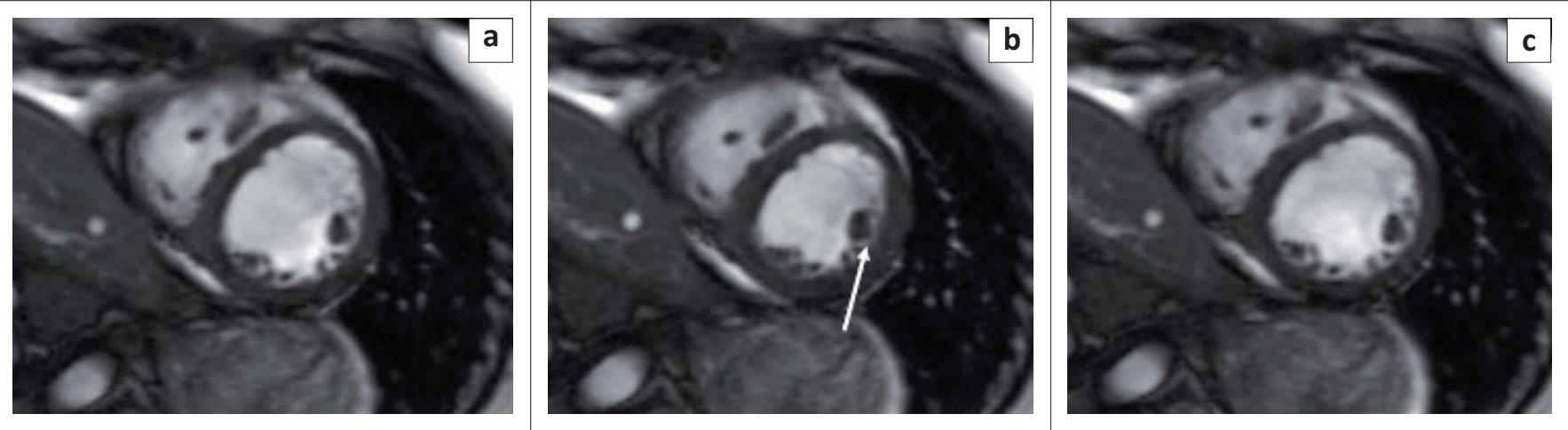

FIGURE 3: Three short-axis cine SSFP images of the LV at the phases ( $a, b$ and $c$ ) close to end-systole. When selecting the end-systolic and end-diastolic phases, it is advisable to observe the relationship of the LV wall to the papillary muscles. When the myocardium is closest to the papillary muscle (white arrow), just before it starts moving away from the LV wall again, is the end-systolic phase. When the lateral wall is furthest away from the papillary muscles, just before it starts moving towards the LV wall again, that is the end-diastolic phase. For video footage, visit: https://www.youtube.com/watch?v=LTbqVPUYGUo\&feature=youtu.be.

\section{Right ventricular function}

RV function plays a particularly large role in the assessment of patients with congenital heart disease, and serial assessments of RV function are used to determine the timing of surgical interventions. CMR has now emerged as the reference standard for this assessment owing to its accuracy and reproducibility. ${ }^{5}$

The same contouring principles used for LV post-processing apply for the contouring of RV functional assessments: play the cine movie initially; delete any repeat slices; select the 


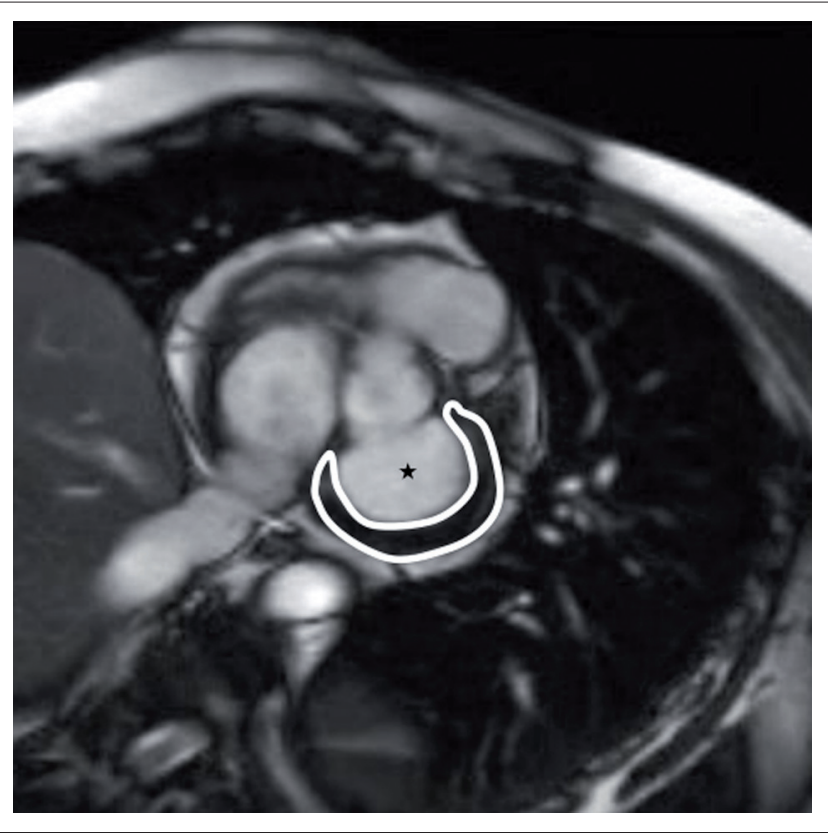

FIGURE 4: Single short-axis cine SSFP image of the basal LV myocardium. The basal slice is the first slice where the blood pool (*) is encircled by more than 180 degrees of myocardium (white contour).

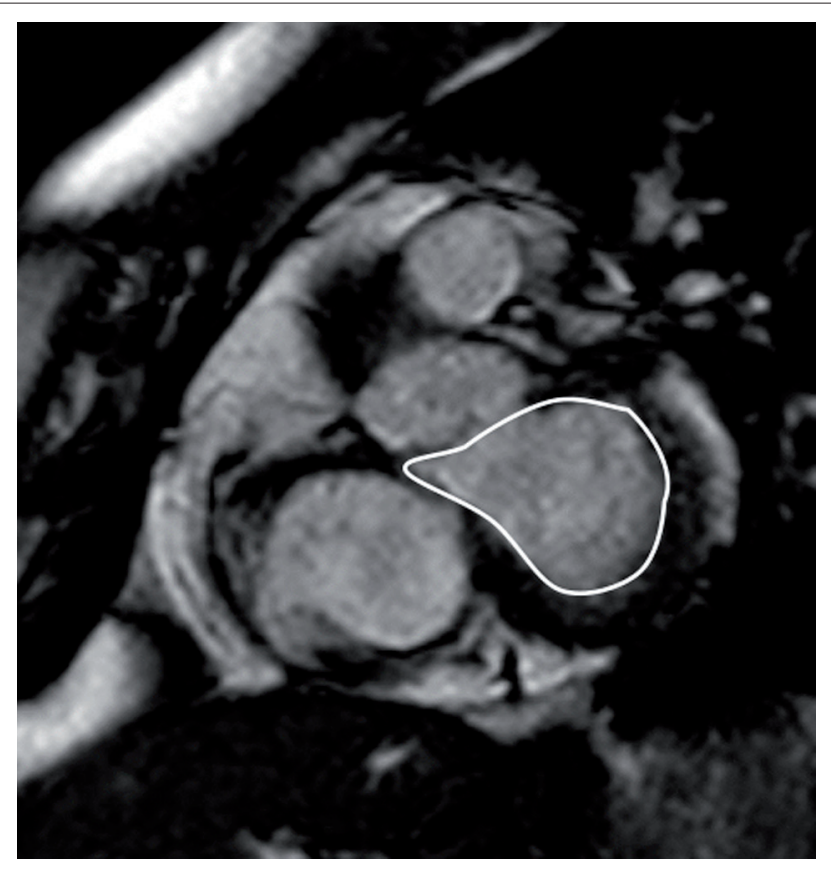

FIGURE 5: Single short-axis cine SSFP image of the basal LV myocardium. The left ventricular outflow tract should be included in the blood pool when contouring the LV endocardium contour up to the level of the aortic valve leaflets.

end-systolic and end-diastolic phases; and contour the endocardial border. Only the RV endocardial border is contoured as the RV myocardium is very thin, and RV myocardial mass is usually only important in research settings.

The RV can be contoured on the short-axis cine images (Figure 7), which is the same stack of images used for LV contouring, or on the transaxial cine images (Figure 8). Because both these sequences have their own advantages and disadvantages, the Task Force guideline does not specify which one to utilise. The biggest advantage to using

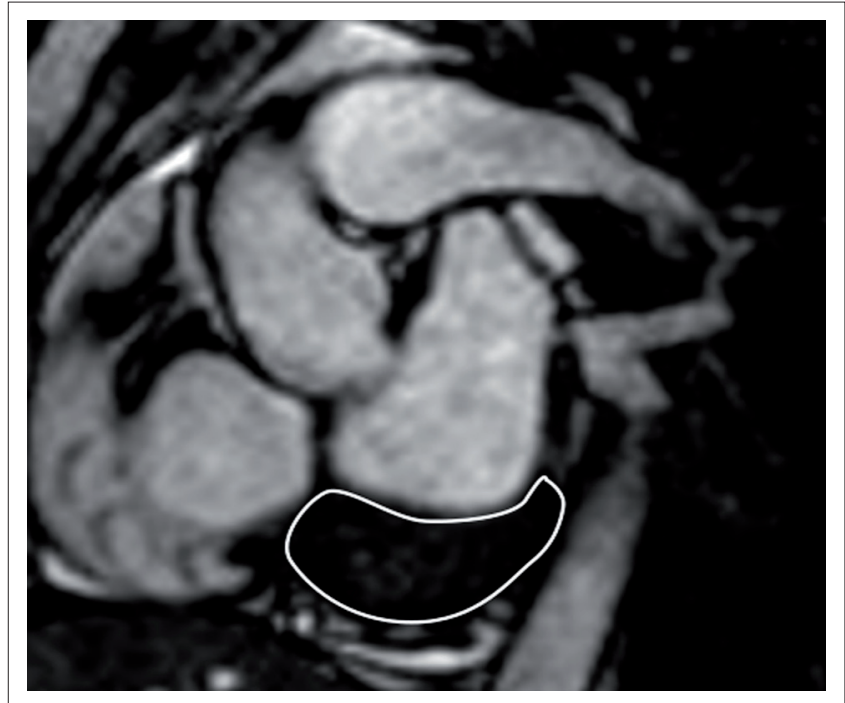

FIGURE 6: Single short-axis cine SSFP image of the basal LV myocardium. If only a small portion of the LV myocardium is evident in the most basal slice with no discernible LV blood pool, then only the epicardial border is contoured for the LV mass assessment.

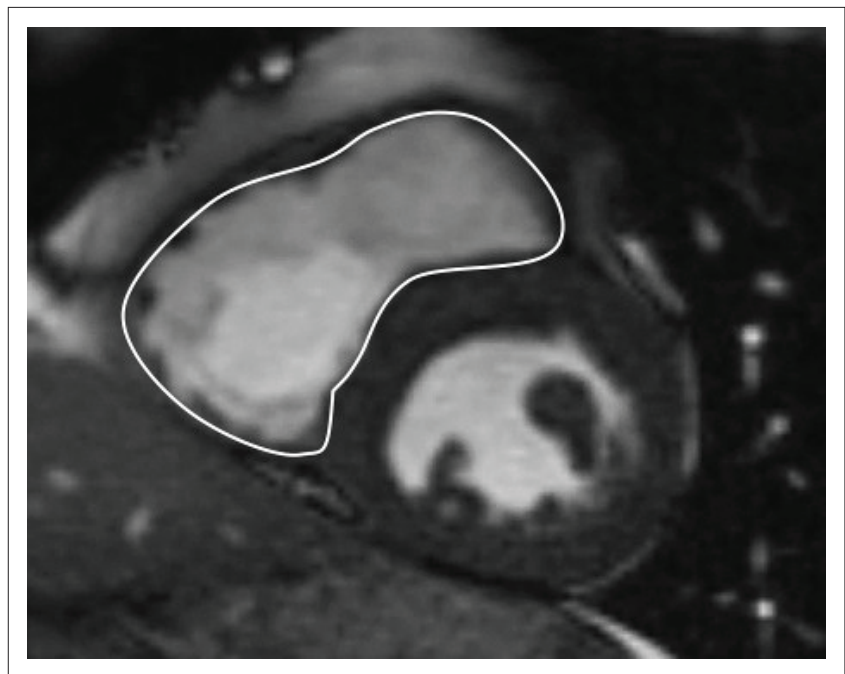

FIGURE 7: Single short-axis cine SSFP image at the mid-ventricular level. The RV is contoured on the stack of short-axis cine images (white line).

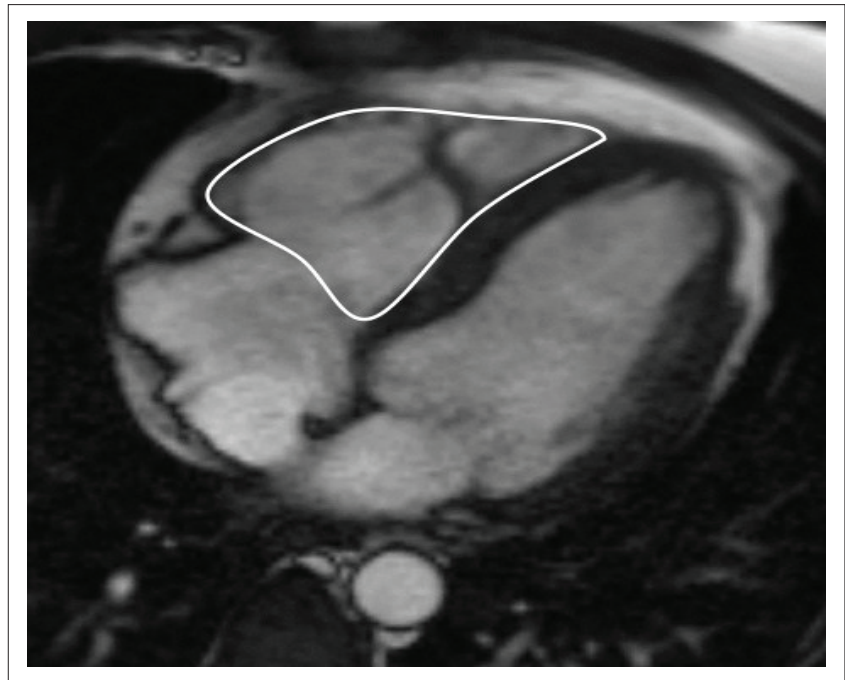

FIGURE 8: Transaxial cine SSFP image of the heart. The levels of the tricuspid and pulmonary valves are easily visualised on the transaxial images for the contouring of the RV volumes and function (white line). 
the short-axis view is that it is the same sequence used for the LV function, which saves time. The disadvantages are that it is often difficult to choose the basal slice on the shortaxis stack, and that the pulmonary valve is normally not well visualised. The pulmonary and tricuspid valves are easily observed on the transaxial images which defines the ventricular borders clearly, but this means that an extra data stack must be acquired. The RV endocardial border is drawn as a smooth line at the inner margin of the RV myocardium. The moderator band should be included in the blood pool unless it is exceptionally large, in which case it can be excluded.

Tip: If there is no cardiac shunt, the stroke volume of the LV should roughly match that of the RV.

\section{Atrial size measurement}

The atria are measured during ventricular systole when the mitral valve is closed (Figure 9). There are a number of ways
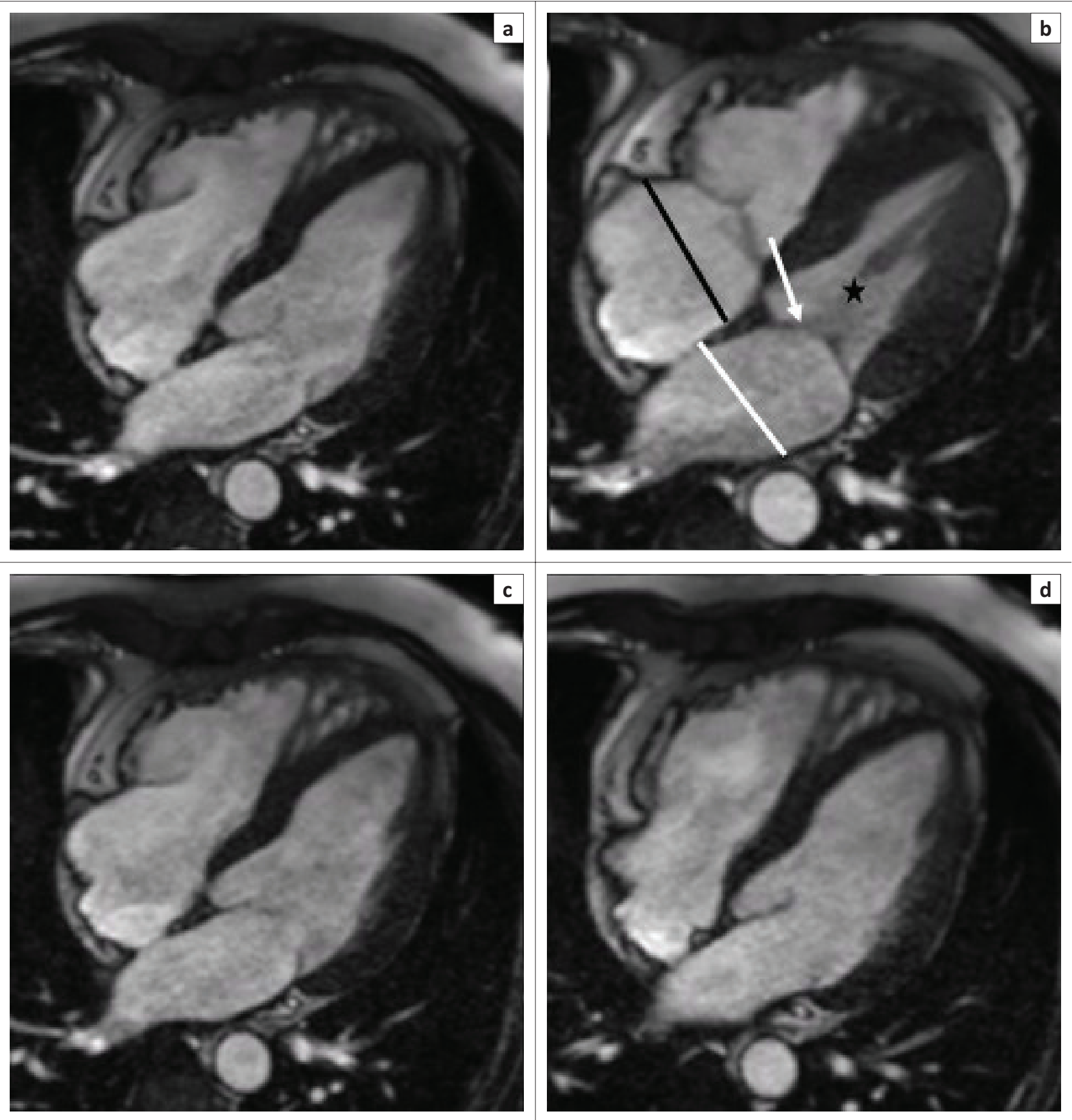

FIGURE 9: Four-chamber cine SSFP images (a-d) of the heart. The atria are measured at the end of the ventricular end-systole when the ventricle blood pool is the smallest $(*)$ and the mitral valve is closed (white arrow). The atria can be measured with a transverse measurement at the level of the mid atrium (right atrium - black line; left atrium - white line), avoiding the atrial appendages and pulmonary vein orifices. 
to measure the atria, and the importance of obtaining the exact size of the atria will determine which of these are used. Routine measurements are normally done as a transverse measurement of the maximum diameter of the atrium, taking care to exclude the ostia of the pulmonary veins and the appendages (Figure 9). Normal atrium size is less than $4 \mathrm{~cm}$ on this measurement. The atria can also be measured using the biplane area-length method, using a longitudinal measurement from the posterior wall to the mid AV valve as well as a transverse measurement perpendicular to this in the mid atrial level (Figure 10). Lastly, the atria can be contoured like the ventricles for exact volumes.

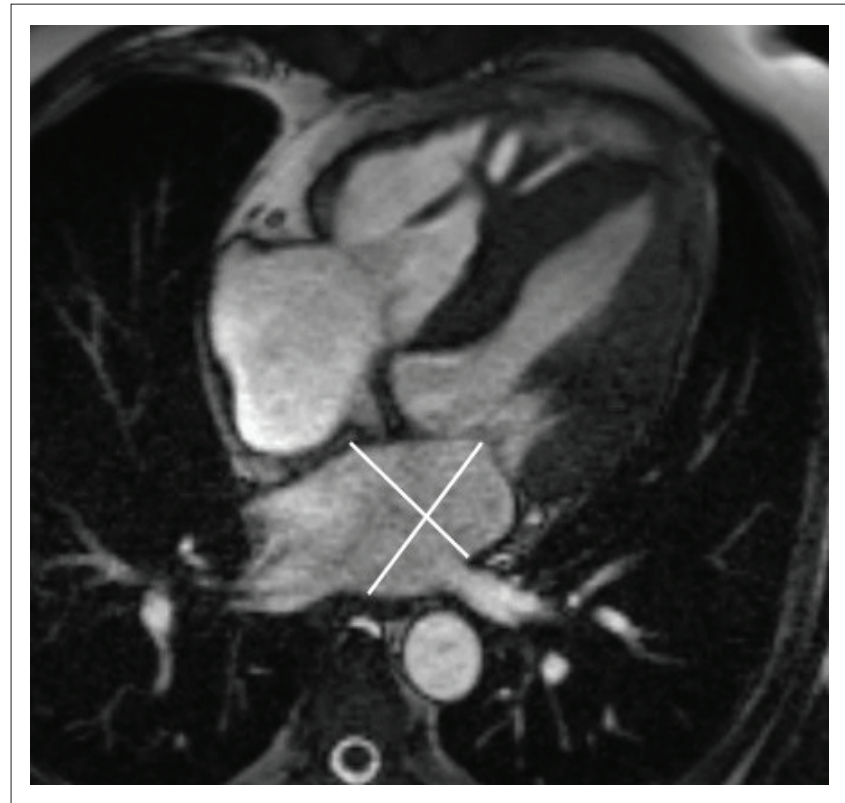

FIGURE 10: Four-chamber cine SSFP image of the heart. The atria can also be measured with the biplanar method with a longitudinal measurement from the mid atrioventricular valve to the posterior atrial wall and a perpendicular measurement transversely through the mid atrium.

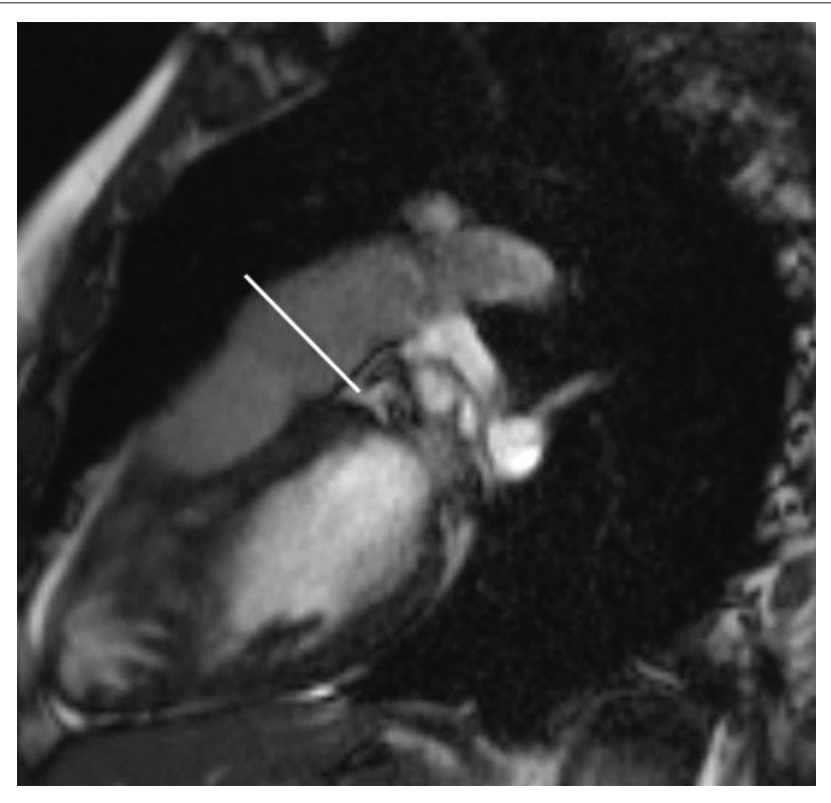

FIGURE 11: Right ventricle outflow tract cine SSFP image. The phase contrast sequence is planned perpendicular to the vessel (white line), in this case the pulmonary artery.

\section{Flow analysis}

Phase contrast analysis currently plays an important part in the functional assessment of patients with valvular and congenital heart disease. ${ }^{6}$ For these values to be reliable, it is important to acquire these sequences correctly. The images need to planned and acquired perpendicular to the vessel in question (Figure 11). Normally, this will be performed for the aorta and the pulmonary artery; this allows calculation of the pulmonary-to-systemic flow ratio (Qp:QS) during shunt assessment. Flow analysis is also used in valvular heart disease for peak velocity and regurgitation fraction assessment. The flow sequence generates two image sets: a magnitude and a phase contrast set (Figure 12).

Tip: The magnitude image set has more anatomical detail and should be used to draw the region of interest (ROI) (Figure $12)$; this can then be propagated to the phase contrast images.
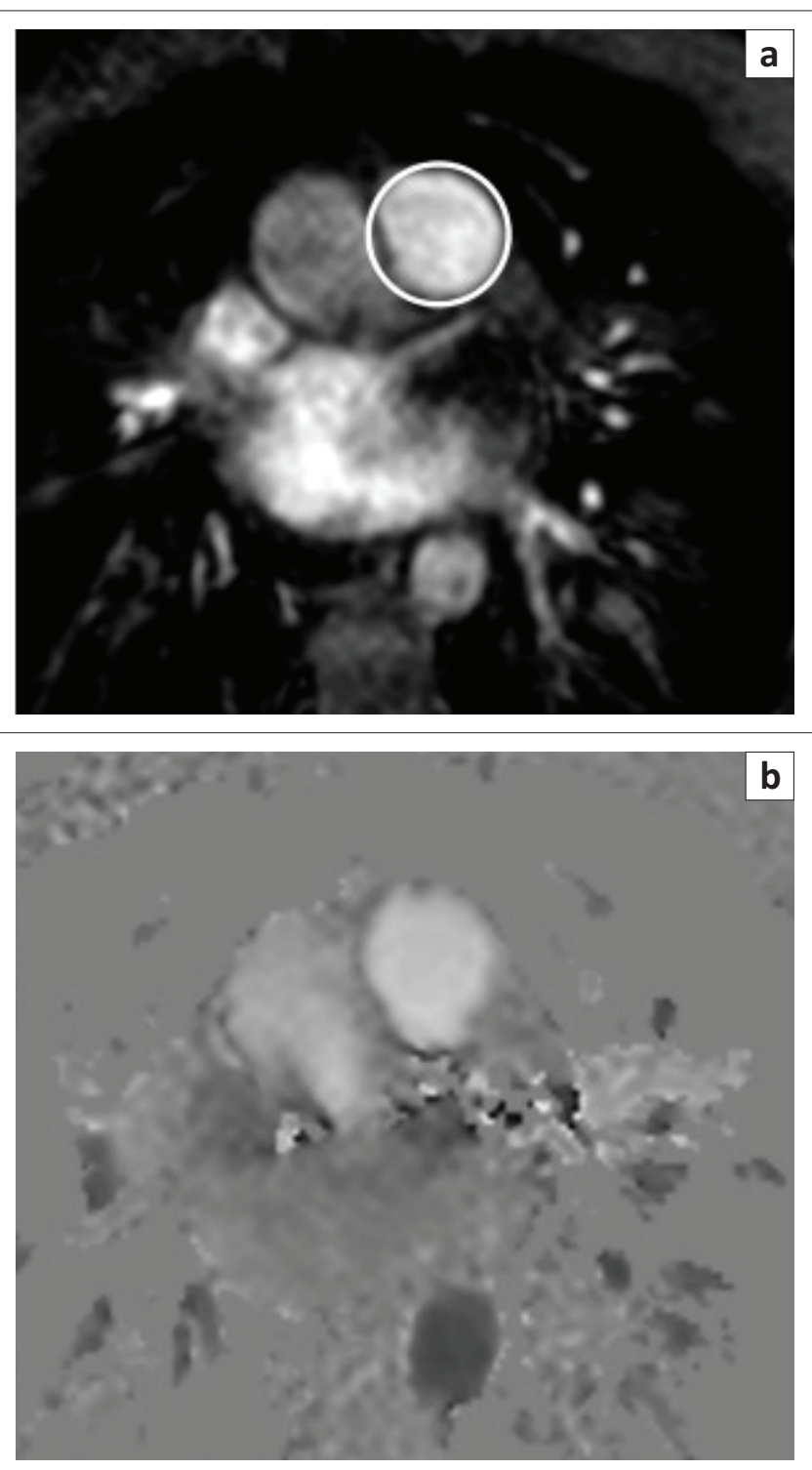

FIGURE 12: Phase contrast images perpendicular to the pulmonary valve. The phase contrast series generates two sets of images: the magnitude image: (a) that clearly defines the anatomical structure, and (b) the phase contrast image that depicts the blood flow with less anatomical detail. When performing contours, it is advisable to draw the ROI on the magnitude image (white contour) and then to propagate them to the phase contrast image. 

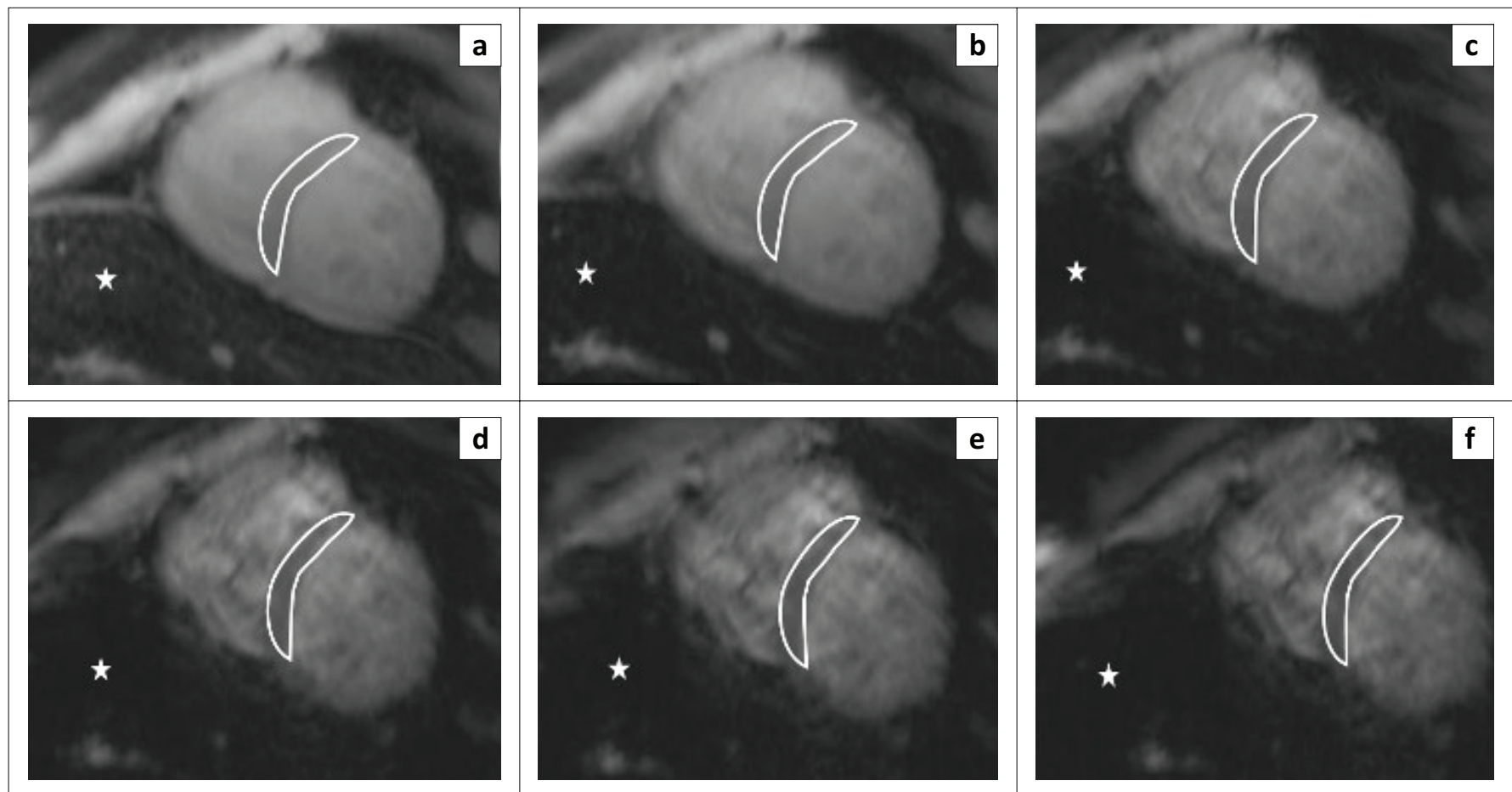

FIGURE 13: Serial short-axis T2* images at the mid ventricular level at different echo times. The ROI for T2* assessment is drawn to include as much of the myocardium as possible, excluding the blood pool in both ventricles. The signal loss in the liver $\left(^{*}\right)$ owing to iron infiltration can also be seen.

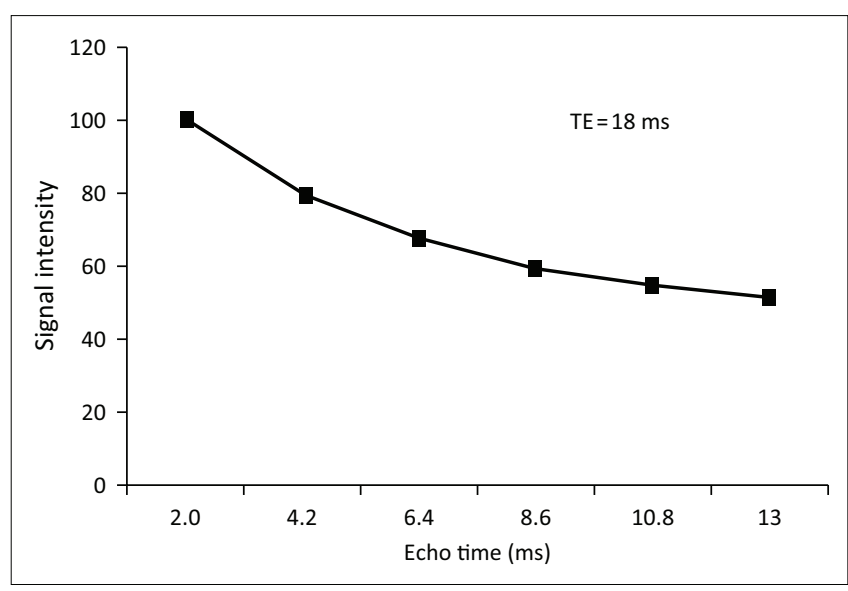

FIGURE 14: Signal intensity to echo time graft of the T2* analysis with the sample points for the ROI at each TE time having a smooth continuous contour.

It helps to be generous with the margins when drawing the ROI to ensure that peripheral flow in the vessel is included. However, care should be taken not to include adjacent vessels as this will influence the flow values.

\section{T2* analysis and aortic measurement}

T2* analysis is used to quantify the amount of iron deposition in the myocardium and is an important diagnostic and prognostic factor for siderotic cardiomyopathy in transfusiondependent patients. ${ }^{7}$ The sequence is performed in the shortaxis orientation and will provide an image set with different time-to-echo (TE) times for the same slice position (Figure 13). The septum is used for the analysis and a ROI is drawn to include as much of the septum as possible, but taking care to avoid the blood pool in both ventricles as well as the anterior and inferior hinge points (Figure 13); this will avoid the blood pool trapped in the myocardial trabeculations in the hinge point regions and also avoid septal perforator arteries. The software will then generate a curve (Figure 14).

Tip: If there is a point on the graft that is an outlier, review the contours for the correlating TE time; usually that results from the inclusion of some of the blood pool in the ROI.

Normal myocardial TE times are more than $20 \mathrm{~ms}$; $15 \mathrm{~ms}$ $19 \mathrm{~ms}$ is considered mild iron infiltration, $10 \mathrm{~ms}-14 \mathrm{~ms}$ is moderate iron infiltration, and $<10 \mathrm{~ms}$ is considered severe iron infiltration. ${ }^{8}$

\section{Aortic measurements}

Aortic measurement levels for vascular assessment have been standardised by the Task Force and are measured at the: sinus of Valsalva, sinotubular junction, mid ascending aorta, distal ascending aorta (proximal to the brachiocephalic artery), arch (between the left common carotid artery and the left subclavian artery), proximal descending aorta (just distal to the left subclavian artery), mid descending aorta, distal descending aorta (at the level of the diaphragm), and abdominal aorta (above the coeliac trunk) (Figure 15).

The aortic valve can be measured either as a sinus-to-sinus measurement (Figure 16) or as a sinus-to-commissure measurement (Figure 17).

\section{Conclusion}

Cardiac MRI is a robust and rapidly developing field that has significantly advanced the management of cardiac patients. It is important that accuracy and consistency be maintained to ensure that we gain and maintain the clinicians' confidence in the use of this modality. 


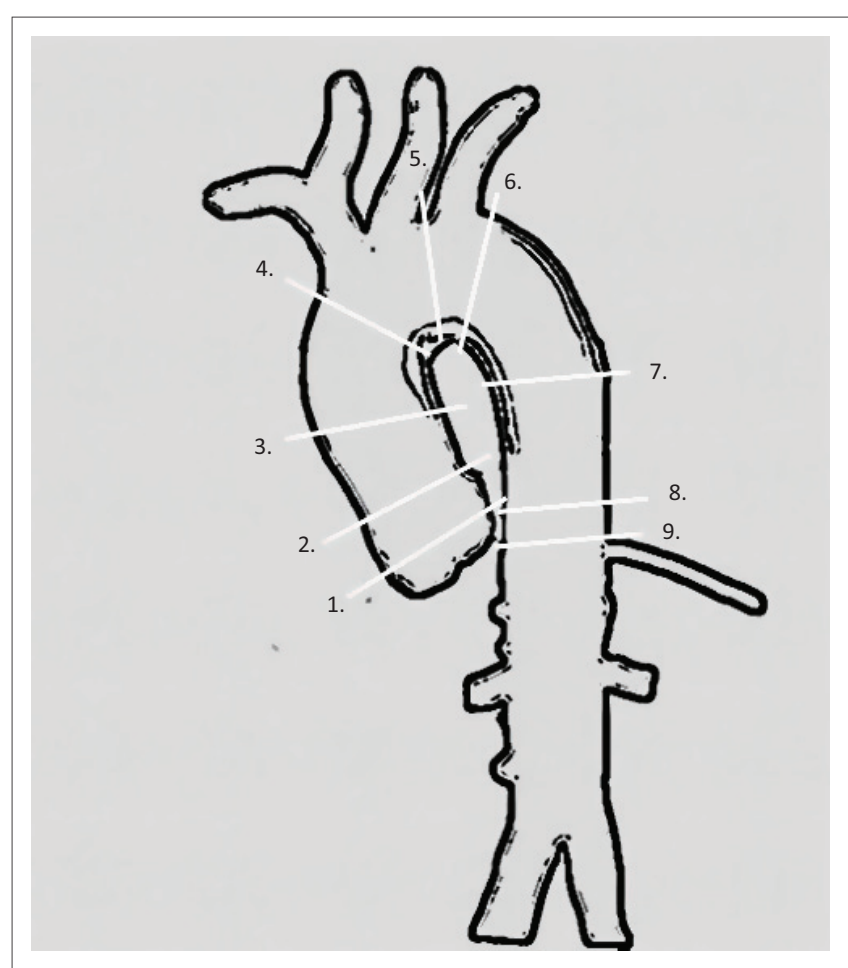

FIGURE 15: Line drawing of the standard aortic measurement positions: Sinus of Valsalva; (2) sinotubular junction; (3) mid ascending aorta; (4) distal ascending aorta (just proximal to the brachiocephalic artery); (5) mid aortic arch (between the left common carotid artery and left subclavian artery); (6) proximal descending artery (just distal to the left subclavian artery); (7) mid descending aorta; (8) distal ascending aorta (at the level of the diaphragm); (9) abdominal aorta (just above the coeliac trunk).

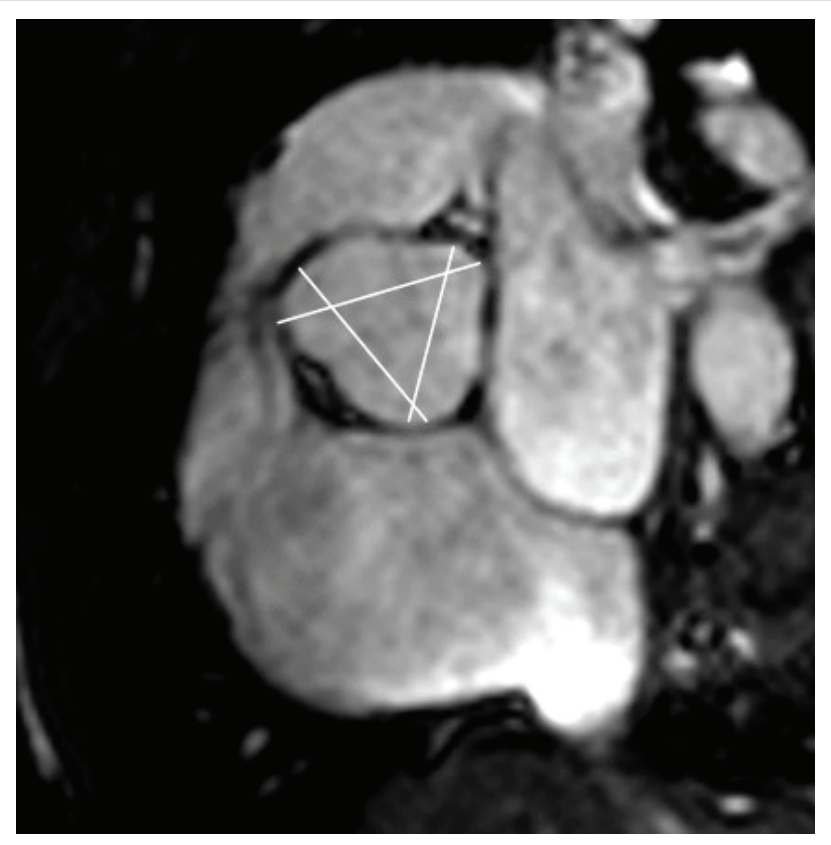

FIGURE 16: Axial cine SSFP image through the aortic valve. One of the methods of measuring the aortic valve is to measure the maximum diameters between the adjacent sinus of Valsalva (white lines) sinus-to-sinus measurement.

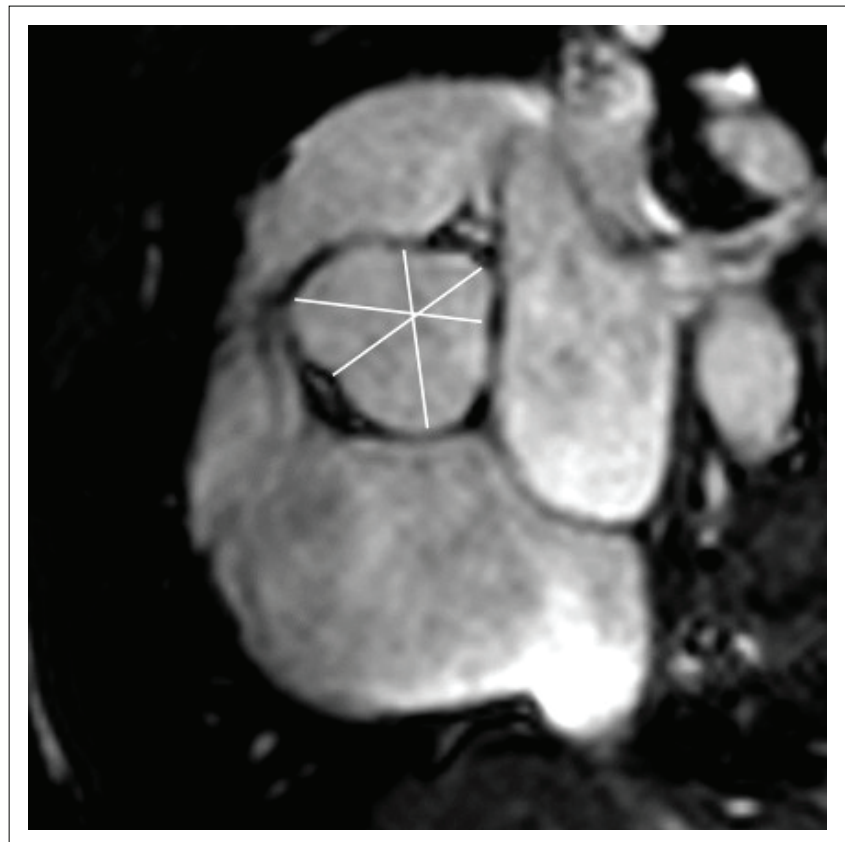

FIGURE 17: Axial cine SSFP image through the aortic valve. The second method of measuring the aortic valve is to measure the distances from the sinus of Valsalva to the opposite commissure (white lines). This is called the sinus-tocommissure measurement.

\section{Acknowledgements Competing interests}

The author declares that she has no financial or personal relationships which may have inappropriately influenced her in writing this article.

\section{References}

1. Flett AS, Westwood MA, Davies LC, Mathur A, Moon JC. The prognostic implications of cardiovascular magnetic resonance. Circ Cardiovasc Imaging. 2009;2(3):243-250. http://dx.doi.org/10.1161/CIRCIMAGING.108.840975

2. Schulz-Menger J, Bluemke DA, Bremerich J, et al. Standardized image interpretation and post processing in cardiovascular magnetic resonance: Society for Cardiovascular Magnetic Resonance (SCMR) board of trustees task force on standardized post processing. J Cardiovasc Magn Reson. 2013;15:35. http:// standardized post processing. J Cardio
dx.doi.org/10.1186/1532-429X-15-35

3. Mekkaoui C, Reese TG, Jackowski MP, Bhat H, Sosnovik DE. Diffusion MRI in the heart. NMR Biomed. 2015. http://dx.doi.org/10.1002/nbm.3426

4. Bellenger NG, Burgess MI, Ray SG, et al. Comparison of left ventricular ejection fraction and volumes in heart failure by echocardiography, radionuclide ventriculography and cardiovascular magnetic resonance; are they interchangeable? Eur Heart J. 2000;21(16):1387-1396. http://dx.doi.org/10.1053/euhj.2000.2011

5. Geva T. Is MRI the preferred method for evaluating right ventricular size and function in patients with congenital heart disease?: MRI is the preferred method for evaluating right ventricular size and function in patients with congenital hear disease. Circ Cardiovasc Imaging. 2014;7(1):190-197. http://dx.doi.org/10.1161/ CIRCIMAGING.113.000553

6. Nayak KS, Nielsen JF, Bernstein MA, et al. Cardiovascular magnetic resonance phase contrast imaging. J Cardiovasc Magn Reson. 2015;17(1):71. http://dx.doi. org/10.1186/s12968-015-0172-7

7. Carpenter JP, He T, Kirk $\mathrm{P}$, et al. On T2* magnetic resonance and cardiac iron Circulation. 2011;123(14):1519-1528. http://dx.doi.org/10.1161/CIRCULATIONAHA. 110.007641

8. Kremastinos DT, Farmakis D. Iron overload cardiomyopathy in clinical practice. Circulation. 2011;124(20):2253-2263. http://dx.doi.org/10.1161/CIRCULATIONAHA. 111.050773 